\title{
Cochlea implantation in patients with superficial hemosiderosis
}

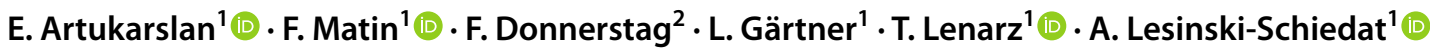

Received: 28 April 2021 / Accepted: 22 November 2021 / Published online: 20 December 2021

(c) The Author(s) 2021

\begin{abstract}
Introduction Superficial hemosiderosis is a sub-form of hemosiderosis in which the deposits of hemosiderin in the central nervous system damage the nerve cells. This form of siderosis is caused by chronic cerebral hemorrhages, especially subarachnoid hemorrhages. The diversity of symptoms depends on the respective damage to the brain, but in most of the cases it shows up as incipient unilateral or bilateral hearing loss, ataxia and signs of pyramidal tracts. We are investigating the question of whether cochlear implantation is a treatment option for patients with superficial hemosiderosis and which strategy of diagnostic procedure has to be ruled out preoperatively.

Materials and methods In a tertiary hospital between 2009 and 2018, we examined $(N=5)$ patients with radiologically confirmed central hemosiderosis who suffered from profound hearing loss to deafness were treated with a cochlear implant (CI). We compared pre- and postoperative speech comprehension (Freiburg speech intelligibility test for monosyllables and HSM sentence test).

Results Speech understanding improved on average by $20 \%$ (monosyllabic test in the Freiburg speech intelligibility test) and by $40 \%$ in noise (HSM sentence test) compared to preoperative speech understanding with optimized hearing aids.

Discussion The results show that patients with superficial siderosis benefit from CI with better speech understanding. The results are below the average for all postlingual deaf CI patients. Superficial siderosis causes neural damages, which explains the reduced speech understanding based on central hearing loss. It is important to correctly weigh the patient's expectations preoperatively and to include neurologists within the therapy procedure.
\end{abstract}

Keywords Superficial hemosiderosis · Cochlear implant $\cdot$ Cochlear spiral ganglion neurons

\section{Introduction}

Superficial hemosiderosis $(\mathrm{SH})$ is a rare progressive disease of the central nervous system (CNS). SH is resulting from subarachnoid bleeding primary build-up of the hemosiderin in the leptomeninges, the cranial nerves, spinal cord and in the subpial tissue [1-6]. The cause of this form of accumulation is chronic bleeding, wherein the hemosiderin acts cytotoxically on the surrounding subarachnoid tissue [5]. The onset of clinical symptoms ranges from 14 to 77 years with a male-to-female ratio of $3: 1[4,7]$. The causes of bleeding are in up to $50 \%$ of the cases idiopathic where no bleeding

E. Artukarslan

artukarslan.eralp-niyazi@mh-hannover.de

1 Department of Otorhinolaryngology, Hannover Medical School, Hannover, Germany

2 Department of Neuroradiology, Hannover Medical School, Hannover, Germany source is found and secondary forms, where a bleeding source can be detected $[4,6,8]$. The main causes of bleeding are head trauma, aneurysm, neoplasm and neurosurgical procedures [5]. In a variably number of superficial siderosis a intraspinal fluid-filled collection of variable dimensions is found [8]. Symptomatically, the SH manifests itself in more than $95 \%$ of cases with uni- or bilateral sensorineural hearing loss (SNHL), in $88 \%$ of cases with cerebellar ataxia and in $76 \%$ of cases with pyramidal signs. Other symptoms include dementia, anosmia and anisocoria in $24 \%, 17 \%$ and $10 \%[2,9]$. For diagnosis of $\mathrm{SH}$, the magnetic resonance imaging (MRI) is to date the gold standard (Fig. 1). The gradient-echo T2* and spin echo T2-weighted are the most sensitive sequences for hemosiderin detection in the CNS marked as low-density regions $[6,10]$.

Pathophysiologically, the Bergmann glia absorbs the iron from the CNS. Stored in the form of ferritin, the hemosiderin is then broken down. Once the binding capacity has been exhausted, the iron ions accumulate and due to the 
Fig. 1 Axial T2*-weighted MRI of Pat XX with superficial siderosis reveals diffuse hypointensity outlining the cortex and nerval tissue in the posterior fossa. A Siderosis of the cranial nerves VII, VIII, IX from their pontine emergence up to the entry in the inner auditory canal (white arrows) and siderosis the cerebellar cortex (white arrow heads). B Siderosis of the quadrigeminal plate (double white arrows) and the upper cerebral vermis (white arrow heads) with sparing of the cortex of both temporal lobes (curved white)

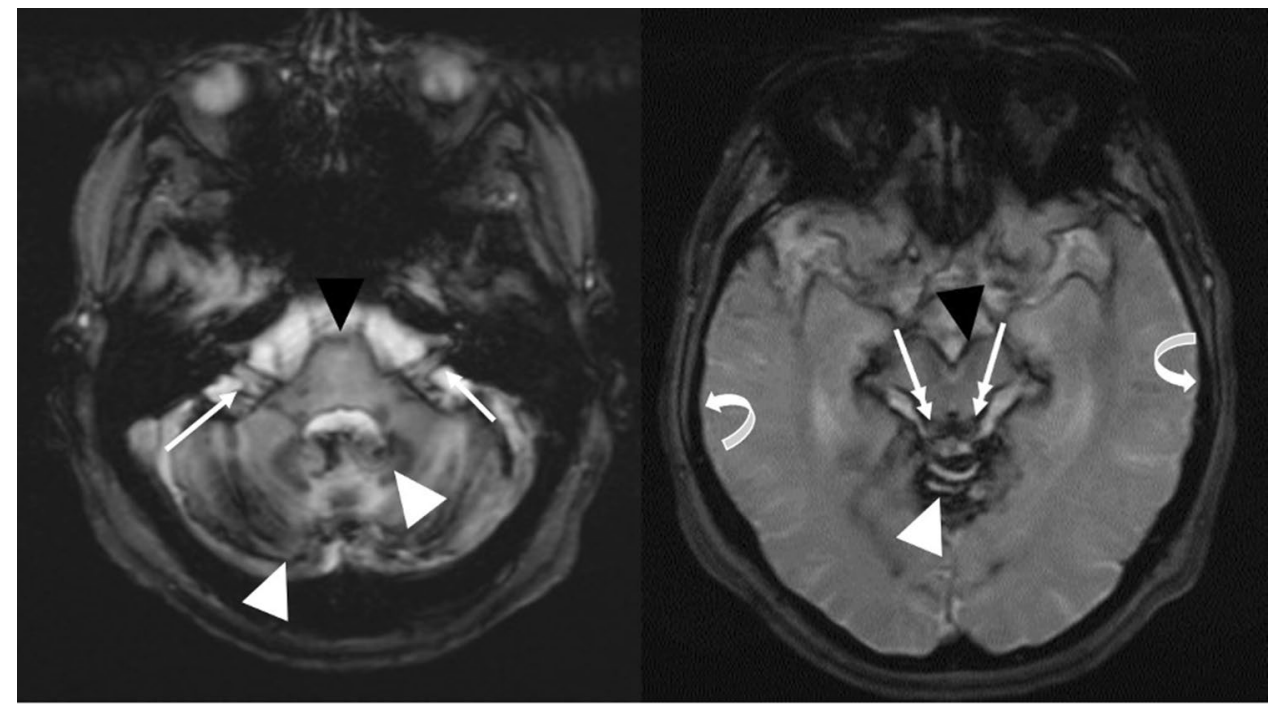

associated lipid peroxidation, parenchymal and axonal damage to the nerves is seen. The cerebellum is a predilection site for the hemosiderin, presumably due to the presence of Bergmann glia in the upper layers. The N. VIII (vestibulocochlearis nerve) is also probably a preferred site because the long course in the pontine cistern and the resulting contact with the hemosiderin is probably higher. In addition, the $\mathrm{N}$. VIII has a long glial segment and is therefore more vulnerable to the accumulation of hemosiderin [4, 5, 7].

The treatment of SH depends on the cause and remains challenging. First of all, bleeding sources should be detected and treated with surgical ablation [5]. If the origin of the SH despite extensive imaging work-out remains unknown a medical treatment with iron chelators could be considered [11]. Due to the frequent finding of intraspinal fluid-filled collection and their associations with intracranial hypotension $[8,12]$, an epidural blood patching may be an individual therapy option [13]. Under computer tomographic (CT) scan guidance, an 18-gauge Tuohy needle is inserted into the epidural space via a median or paramedian interlaminar approach. The epidural position of the needle is confirmed with an injection of 1-2 $\mathrm{ml}$ of iodine containing contrast media and subsequently, patient's blood is injected through the needle. A second CT scan confirms the peridural distribution of the blood in the spinal canal. The patient remains in prone position for at least four hours and bedrest up to the next day.

While uni- or bilateral SNHL affects about $95 \%$ of patients with $\mathrm{SH}$, hearing aids (HA) are means of choice for mild to moderate SNHL. In cases of progressive SNHL to severe-profound hearing loss (HL) where acoustic amplification is no longer sufficient, cochlear implantation (CI) is indicated. Despite retrocochlear genesis of the HL, the literature describes in the majority of cases a sufficient outcome in hearing with CI with 44 cases $[6,9,14-17]$.
Between 2009 and 2018, ten patients with radiologically confirmed central hemosiderosis presented at our tertiary care hospital. Of these ten patients, five cases who suffered from profound hearing loss to deafness were treated with a cochlear implant (CI), four uni- and one bilaterally (Fig. 2). Another two patients with profound hearing loss decided against a treatment with cochlear implants and another two patients with hemosiderosis were diagnosed with a moderate hearing loss and could be fitted with hearing aids. The last patient out of the ten known cases with a profound hearing loss underwent the blood patch therapy and showed a slow progression of his hearing loss.

In this retrospective study, we showcase five patients who were treated with CI. One patient was blood patched before the CI. We report about the CI outcomes of five patients and whether the blood patch therapy had an influence on the $\mathrm{SH}$ symptoms. Based on pre-, intra- and postoperative measurements, we analyze the genesis of the HL.

The ethic approval for this retrospective study was pronounced positively from the responsible ethics committee (number 1897-2013).

\section{Case presentations}

\section{Case 1}

A 68-year-old woman was referred to our clinic in 2012 with deafness on the right and profound SNHL on the left side, ataxia and vertigo. She reported about gradual progressive HL for six years and insufficient acoustic amplification using HA. Cognitive deficits, pyramidal signs or other symptoms associated with $\mathrm{SH}$ were not detected. She had a history of cerebellar infarction in 2006. In 2011, the diagnosis of 

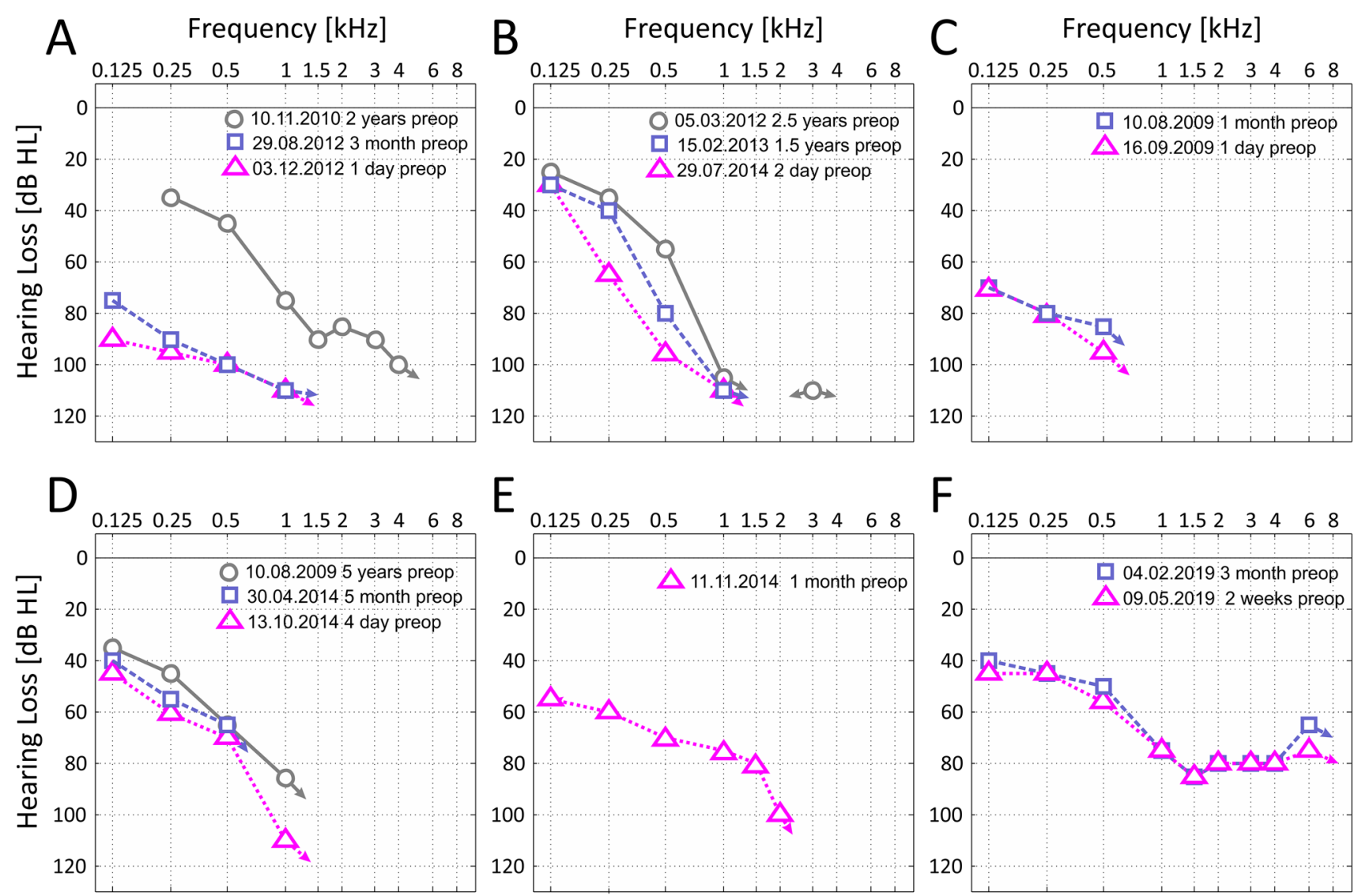

\section{$\mathrm{F}$}
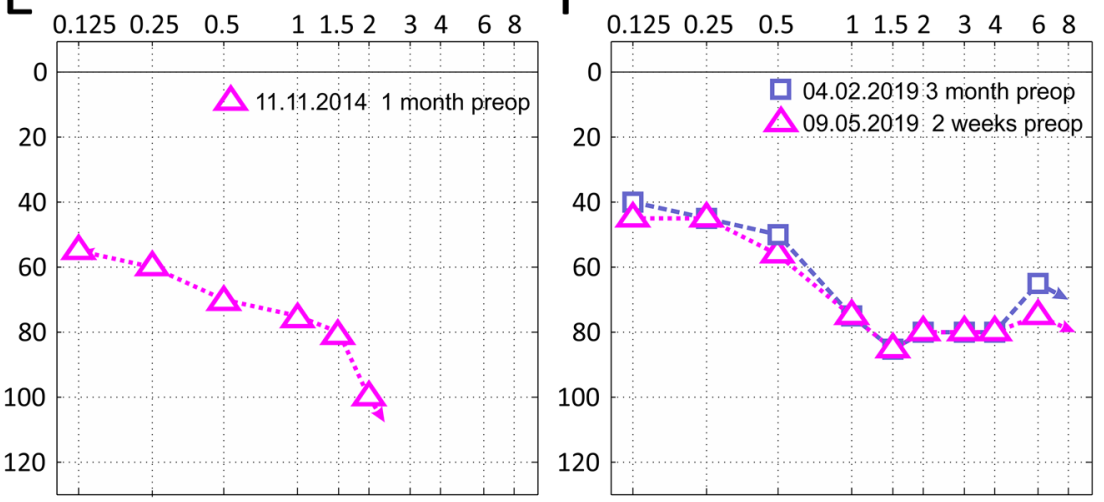

Fig. 2 The figure shows preoperative audiograms of all our five patients. A Case 1; B Case 2; C Case 3 left side; D Case 3 right side; E Case 4; F Case 5

SH and cerebellar worm atrophy was detected with MRI. In angiography, no source of bleeding could be detected.

The audiological and neurootological evaluation revealed (Table 1) deafness on the right side and profound SNHL in the high frequencies and residual hearing in the low frequencies on the left side. In the Freiburg Monosyllabic Word Test (FMWT) in quiet at $65 \mathrm{~dB}$ sound pressure level (SPL) under best aided condition, she showed $0 \%$ recognition on the right side and $10 \%$ recognition at $80 \mathrm{~dB}$ on the left side. Auditory brainstem response (ABR) did not record any responses at maximum intensity of $95 \mathrm{~dB} \mathrm{nHL}$ on both sides. Transient evoked otoacoustic emissions (TEOAE) were not present bilaterally. The transtympanic promontory electrical stimulation (tt-PES) elicited a sound sensation on both sides. The vestibular examination showed a canal paresis on the left side in the caloric test and a vestibular and visual deficit in the posturography. The CT of petrous bones showed no abnormalities. The CI surgery was conducted in 2012 on the right side with a CI24RECA electrode that was fully inserted using a round window approach (RWA). Intra- and postoperatively, no complications were reported.
Intraoperatively, electrically evoked compound action potential (ECAP) and electrically evoked stapedius reflexes (ESR) were measured using the clinical software CustomSoundEP. ECAP responses were successfully recorded via the AutoNRT (automatic neural response telemetry) task over the whole electrode array with regular morphology. Also, ESR were elicited successfully. The Hochmair-Schulz-Moser test (HSM-Test) showed no speech understanding pre- and postoperative. ECAP responses were still detectable on each of the 22 electrode contacts. All AutoNRT traces were checked according to Gärtner et al. [18]. Only minor corrections were necessary. ECAP thresholds were found in between 174 and 210 $\mathrm{CL}$ (at $25 \mu$ s pulse duration) corresponding to 10.1 and $19.4 \mathrm{nC}$, respectively. The slope of the ECAP amplitude growth function (AGF) was mostly below $1 \mu \mathrm{V} / \mathrm{CL}$, and hence very shallow comparing to normal CI users. Postoperatively in the follow-ups up to 13 months, the FMWT showed no improvement in speech recognition with CI. 


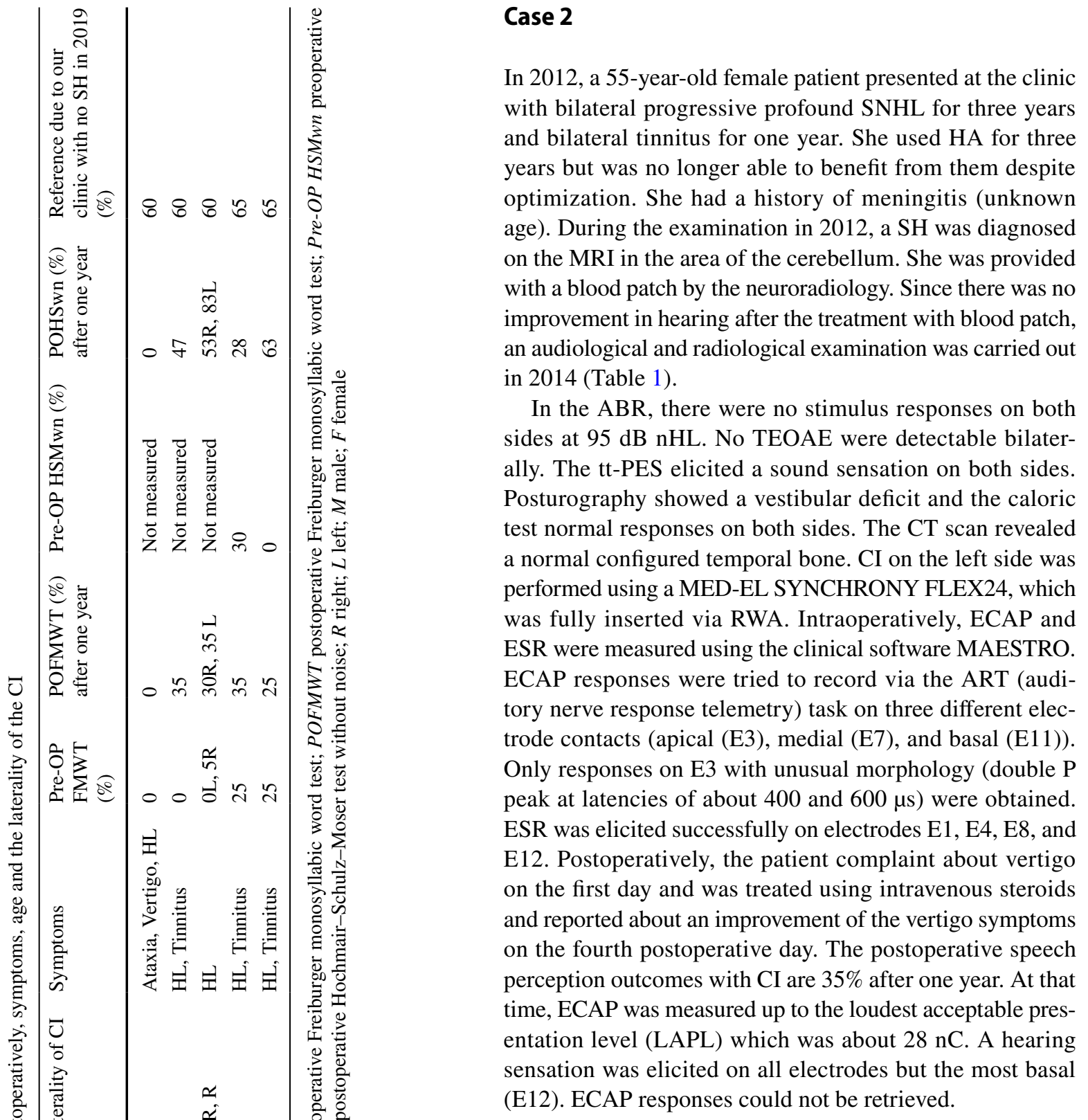

Case 3

In 2009, a 59-year-old male patient was referred to the clinic with a progressive SNHL on both sides since 1970. Other otological symptoms, cerebellar ataxia, pyramidal signs were not reported. He was insufficiently fitted with a HA on the left side. He reported about a peripheral schwannomatosis located on his shoulder. MRI showed SH in the area of the lamina tecti and the cerebellar upper worm, no vestibular schwannoma on both sides.

The CI pre-examination took place in the same year and is partly listed in Table 1 . The ABR showed no response on both sides at $90 \mathrm{~dB}$ nHL. There were no TEOAE on both sides and the tt-PES elicited a sound sensation on both sides. Posturography showed a vestibular deficit and the caloric 
test a hyporesponsiveness on the right side without nystagmus. The CT examination showed a regular temporal bone. The patient received a CI on the left in 2009. The MED-EL SYNCHRONY FLEX28 electrode was fully inserted via RWA. There were no complications intra- and postoperatively. The intraoperative ART and ESR were normal. During the six-month follow-up, the patient's speech understanding with CI improved to $70 \%$ in FMWT in quiet at $65 \mathrm{~dB}$ SPL dB on the left.

During the follow-ups, the patient had a progression of the SNHL in the low frequencies on the right side. In 2014 the audiological examination revealed deafness in the high frequencies and profound SNHL in the low frequencies and the patient asked for a CI on the right side. Since 2009, the patient did not develop new otological symptoms, cerebellar ataxia or pyramidal signs. Except for a canal paresis on the right side in the caloric test, the audiological examination revealed no new findings. He received a CI MED-EL SYNCHRONY FLEX28 on the right with full insertion via RWA. There were no complications intra- and postoperatively. The ART and ESR were normal.

In 2016, the patient complained about pain above the CI device on the right side and tinnitus on the right side. Based on these complaints he did not wear the CI on the right. In addition, he reported about increasing gait and posture insecurities for a few months. The patient had a speech recognition score of $30 \%$ on the right ear, $45 \%$ on the left ear and $35 \%$ on both ears in FMWT at $65 \mathrm{~dB}$ SPL. A soft tissue contact of the CI electrode at the mastoid cavity was seen in the CT imaging. A CI revision on the right was performed in 2017 without any intraoperative complications. Intraoperative ART measurements did not show any ECAP response on the electrodes chosen for stimulation $(\mathrm{E} 1,3,7,11)$. The reason for that was clipping of the implant amplifier at relatively low stimulation charges, $20 \mathrm{nC}(\mathrm{E} 3), 30 \mathrm{nC}(\mathrm{E} 7)$, and $36 \mathrm{nC}$ (E11). For the sake of time, recording parameters were not further optimized. ESR measurements were not performed intraoperatively. During the initial fitting, one month after surgery, ART did not reveal responses up to $28.5 \mathrm{nC}$ (LAPL). MCLs were between 19 and $28 \mathrm{nC}$. The CI outcomes of both sides are $30 \%$ on the right and $35 \%$ on the left side after one year.

\section{Case 4}

In 2014, a 73-year-old female patient with progressive SNHL on both sides for seven years and tinnitus for four years was referred to the clinic. No other otological symptoms were reported. She was provided with HA on both sides since 2007 without benefit at the time of presentation. Due to her polyarthrosis of the hip and knee joints, she had gait insecurities. The MRI showed a SH with punctum maximum on the cerebellum upper worm and tectum mesencephali left with affected nerve structures of the internal auditory canal and cerebellum bridge angle.

The audiogram showed profound SNHL on the right and deafness on the left side (Table 1). In the ABR, there were no stimulus responses on either side up to $95 \mathrm{~dB} \mathrm{nHL}$ and no TEOAE on both sides. The tt-PES elicited a sound sensation on both sides. No posturography was performed because of the patients' polyarthrosis. The caloric test showed normal responses on both sides. The CT examination showed regular structures of the temporal bone. The CI on the left (MED-EL SYNCHRONY FLEX28) was fully inserted via RWA in 2014. Intraoperatively, ECAP responses were tried to record via the ART task on seven different electrode contacts (E1, E2, E5, E7, E8, E9, E10). Up to a stimulation level of $36 \mathrm{nC}$, no response was obtained. Neither, ESR could be elicited up to $35 \mathrm{nC}$ on electrodes E1, E4, E8, E11, and E12). No complications were reported intra- and postoperatively. Initial fitting was done six weeks after CI. Hearing sensation was elicited on each of the 12 electrode contacts. At that time, no ECAP responses could be recorded up to the LAPL of about $28 \mathrm{nC}$. The speech understanding outcomes with CI are $35 \%$ after one year.

\section{Case 5}

A 56-year-old male patient with Marfan syndrome was referred to the clinic in 2019 with bilateral progressive SNHL and tinnitus since 2015. Despite an initial benefit from HA use since 2015 and regular optimizations the prosthesis did no longer provide sufficient acoustic amplification. He reported no other otogenic symptoms. No histories of head trauma, neurosurgical procedures or chronic bleeding were reported.

During the CI pre-examination in 2019, a MRI revealed a SH in the subarachnoid space.

The audiogram showed a moderate to profound SNHL in the right ear. A moderate SNHL in the low frequency range and a profound SNHL in the middle and high frequency range were shown on the left side (Table 1). At $95 \mathrm{~dB} \mathrm{nHL}$, the ABR showed no stimulus responses on either side. The TEOAE were not present on both sides. The tt-PES was only testes on the left and elicited a sound sensation. The posturography revealed a somatosensitive and vestibular deficit. The caloric test showed are flexia on both sides. Based on the results, he was implanted on the left in 2019 with a MED-EL FLEX26 electrode with full insertion via RWA. ECAP measurements were performed using the AutoART task within the clinical software MAESTRO and responses were observable on different electrode contacts (E2, E4, E7) with normal morphology and latencies. ESR was tried to elicit without success on electrodes E1, E4, E8, and E12 up to a stimulation level of about $40 \mathrm{nC}$. There were no complications intra- and postoperatively. At initial fitting, 
one-month postop, regular ECAP responses were recordable on all 12 electrode contacts via AutoART. ECAP AGF slope was very shallow ranging between 4 and $17 \mu \mathrm{V} / \mathrm{nC}$. The one-year follow-up speech comprehension results are $25 \%$.

\section{Discussion}

$\mathrm{SH}$ is a rare disease of the CNS with an estimated incidence of $0.15 \%$ [19]. In most cases, SH is diagnosed by coincidence due to its cardinal symptom of bilateral progressive SNHL and disproportionately bad speech understanding with hearing aids because of the progressive neural damage. The literature describes symmetrical HL, beginning with the loss of high frequencies and progressing over all frequencies $[6,14,20,21]$. The progression of symptoms especially HL varied in our patients, while patient no. five had deafness in the left ear within one year, patient no. three reported HL that had been slowly progressing since the 1970s (Table 1). In our series of patients, the majority showed a bilateral severe to profound HL with rapid progression to at least unilateral deafness on one ear disproportionately bad and speech understanding with hearing aids. Three patients in our case series reported about tinnitus as another otogenic symptom and vestibular deficits were seen in the preoperative posturography of most of our patients. Cerebellar ataxias were neither historically nor clinically diagnosed in our patients. The third cardinal symptom that is associated with SH—pyramidal tract signs—could not be observed in our patients.

So far, there is no definitive therapy for SH. The onset of symptoms after bleeding is reported in the literature as 4 months -37 years $[1-3,20]$. The therapy consists primarily in finding possible causes of bleeding and hemostate them and minimizing risk factors that increase the likelihood of bleeding in the sense of a lifestyle change is indicated anytime. River et al. showed a reduction of the iron in the cerebrospinal fluid (CSF) with the treatment of trientine ( $2 \mathrm{~g} / \mathrm{die})$, a chelating agent which is primarily used for the treatment of Wilson with intolerance to D-penicillamine. The drug can cross the blood-brain barrier and, according to the source, slowing down the disease [20].

The treatment with trientine is recommended to patients with a history of brain surgeries or known sources of bleeding, i.e., patients with the secondary form of hemosiderosis.

All patients in our case series were considered to have a primary form of SH because no source of bleeding could be determined. Therefore, none of our patients were treated with trientine.

In the current literature, there are no cases addressed with blood patching in the sense of individual therapy for $\mathrm{SH}$, but in the treatment of intracranial hypotension with hemorrhagic spinal epidural fluid collections [22]. According to this hypothesis, one of our five patients was treated with a spinal epidural blood patch as an individual therapy approach. The individual treatment approach of a blood patch therapy presupposes annual follow-ups with cranial MRI scans. So, the patient underwent yearly MRI controls and chose to be implanted with a CI in 2014 due to the rapidly progressing HL. According to the patient and as shown in the audiometric measurements, the HL did not slow down by this therapy. In contrast, the other patients with profound hearing loss were already in need of a CI and as a result, this therapy became less ideal-as the artifact from the CI on the MRI images would have made it difficult to evaluate the effect of the therapy via imaging. It is currently not possible to make a statement about the therapy results of blood patching after only one described case. But as an alternative, especially with very slow progression of the disease and relatively stable hearing, blood patching therapy can also be considered, with annual MRI evaluation, as a wait and scan therapy.

Although a retrocochlear genesis of SH has previously been considered, Nadol et al. showed in a histopathological study the cochlear involvement manifested by degeneration of the organ of Corti, the spiral ligament and the stria vascularis [23]. Our intraoperative measurements also add support on primary failure of the neural structures within the cochlea in these patients. Preoperatively, no compound action potentials (CAP) were measured by any of the patients. The ECAP AGF slope can be seen as a measure of the density of spiral ganglion cells [24]. Interestingly, we found in our cases a shallow ECAP AGF slope, which may be interpreted as low density of spiral ganglion cells (SGC). Or, in other cases, we did not succeed retrieving ECAP responses at all before the patient complained excessive loudness. This may also be attributed to low SGC density since responses of many SGNs are necessary to record an evoked potential.

Different outcomes in patients with SH using CI are described in the literature review of Taylor et al. as well as observed in the case series of seven patients of Modest et al. [14, 16]. In our case series, three patients showed sustained benefit from their CI in the mean follow-up time of 12 months. The outcome improved on average $26 \%$ in FMWT at $65 \mathrm{~dB}$ SPL. One patient gained no benefit and one showed initial improvement with subsequent decline in speech perception over time so that the progressive nature of the disease was clearly shown. While this patient achieved initially $70 \%$ in the FMWT at $65 \mathrm{~dB}$ SPL after the first CI on the left side in 2009 , six months postoperatively, this value dropped to $45 \%$ in 2014 and then to $30 \%$ in 2018 in the same ear (Table 1). At least a comparison between the speech perception outcomes of the $\mathrm{SH}$ patients with CI with the results of our annual clinic report of the performed implantations between 2014 and 2018 is 
important. It was difficult to provide a long-term follow-up of the presented cases in this study. Some of our patients were lost to follow-up due to health problems caused by $\mathrm{SH}$. The difficulty here is that a low rate of adaptation of the cochlear implants, especially in the case of a progressive disease, can lead to poorer hearing performance. In turn, poor hearing performance can lead to a low level of acceptance for wearing the CI. There are four groups that were categorized according to the preoperative result in the FMWT in our annual report of which we could compare two groups to the case series of SH patients [25]: adult patients with preoperative speech test results of $0 \%$ monosyllable at $65 \mathrm{~dB}$ and 5-20\% monosyllable at $65 \mathrm{~dB}$ with their own HA achieved a median of $60 \%$ and $65 \%$ monosyllable at $65 \mathrm{~dB}$, one year after implantation.

In case 1 , there was no speech understanding at all. In such cases, auditory brainstem implant (ABI) or auditory middlebrain implant (AMI) should be considered as an alternative therapy option for the patient. Knowing that the SH has a retrocochlear genesis, it is important to verify where the damage in the auditory pathway is. Therefore, an audiological and radiological individual diagnosis for each patient is very important. In the future it will be very interesting to localize the damage exactly in the MRI to see how this affects the language understanding after receiving CI. It will also be interesting to verify prognostic factors due to the preoperative MRI.

In summary, it should be noted that early MRI diagnosis in the context of a rapid progressive bilateral SNHL within years, especially with a history of head trauma, aneurysm, neoplasm and neurosurgical procedures is indispensable. If $\mathrm{SH}$ is diagnosed in the imaging frequently, follow-ups should be timed to be able to assess the progression of the disease specially the progression of the leading symptom of HL. Early detection and intervention for HL can have an impact on the outcome with CI. Since the different outcomes with $\mathrm{CI}$ in patients with $\mathrm{SH}$ are uniform throughout the literature, they have to be included in the preoperative patients counseling.

\section{Author contributions Not applicable.}

Funding Open Access funding enabled and organized by Projekt DEAL.

Availability of data and material Not applicable.

Code availability Not applicable.

\section{Declarations}

Conflict of interest The authors declared no potential conflicts of interest with respect to the research, authorship, and/or publication of this article.

Open Access This article is licensed under a Creative Commons Attribution 4.0 International License, which permits use, sharing, adaptation, distribution and reproduction in any medium or format, as long as you give appropriate credit to the original author(s) and the source, provide a link to the Creative Commons licence, and indicate if changes were made. The images or other third party material in this article are included in the article's Creative Commons licence, unless indicated otherwise in a credit line to the material. If material is not included in the article's Creative Commons licence and your intended use is not permitted by statutory regulation or exceeds the permitted use, you will need to obtain permission directly from the copyright holder. To view a copy of this licence, visit http://creativecommons.org/licenses/by/4.0/.

\section{References}

1. Koeppen AH, Dentinger MP (1988) Brain hemosiderin and superficial siderosis of the central nervous system. J Neuropathol Exp Neurol 47(3):249-270

2. Fearnley J, Rudge P (1995) Treatment of superficial siderosis of the central nervous system. Mov Disord 10(5):685

3. Fearnley JM, Stevens JM, Rudge P (1995) Superficial siderosis of the central nervous system. Brain 118(Pt 4):1051-1066

4. Dhooge IJ, De Vel E, Urgell H, Gallego S, Vinck B (2002) Cochlear implantation in a patient with superficial siderosis of the central nervous system. Otol Neurotol 23(4):468-472

5. Berrettini S, De Vito A, Bruschini L, Fortunato S, Forli F (2012) Cochlear implantation in patients affected by superficial hemosiderosis of the central nervous system. Eur Arch Otorhinolaryngol 269(1):25-31

6. Yoo A, Jou J, Klopfenstein JD, Kattah JC (2018) Focused neurootological review of superficial siderosis of the central nervous system. Front Neurol 9:358

7. Bittencourt AG, Goffi-Gomez MV, Pinna MH, Bento RF, de Brito R, Tsuji RK (2012) Programming peculiarities in two cochlear implant users with superficial siderosis of the central nervous system. Eur Arch Otorhinolaryngol 269(5):1555-1563

8. Kumar N (2010) Neuroimaging in superficial siderosis: an indepth look. AJNR Am J Neuroradiol 31(1):5-14

9. Omichi R, Kariya S, Maeda Y, Nishizaki K (2016) Cochlear implantation is a therapeutic option for superficial siderosis patients with sensorineural hearing loss. J Laryngol Otol 130(4):408-411

10. Sugimoto H, Ito M, Hatano M, Yoshizaki T (2012) Cochlear implantation in a patient with superficial siderosis. Auris Nasus Larynx 39(6):623-626

11. Flores Martin A, Shanmugarajah P, Hoggard N, Hadjivassiliou M (2021) Treatment response of deferiprone in infratentorial superficial siderosis: a systematic review. Cerebellum 20(3):454-461

12. Cheng CY, Chen MH, Wang SJ, Lin KP (2011) A proposed mechanism of superficial siderosis supported by surgical and neuroimaging findings. Med Hypotheses 76(6):823-826

13. Wilson D, Chatterjee F, Farmer SF, Rudge P, McCarron MO, Cowley P et al (2017) Infratentorial superficial siderosis: classification, diagnostic criteria, and rational investigation pathway. Ann Neurol 81(3):333-343 
14. Tyler GK, Martin TP, Baguley DM (2012) Systematic review of outcome of cochlear implantation in superficial siderosis. Otol Neurotol 33(6):976-982

15. Alshehabi M, Walshe P, Viani L (2019) Cochlear implantation in the presence of superficial siderosis. Clin Otolaryngol 44(6):1166-1169

16. Modest MC, Carlson ML, Wanna GB, Driscoll CL (2015) Cochlear implantation in patients with superficial siderosis: seven cases and systematic review of the literature. Otol Neurotol 36(7):1191-1196

17. Chaudhry A, Chaudhry D, Muzaffar J, Crundwell G, Monksfield $P$, Bance M (2020) Outcomes of cochlear implantation in patients with superficial siderosis: a systematic review and narrative synthesis. J Int Adv Otol 16(3):443-455

18. Gartner L, Lenarz T, Joseph G, Buchner A (2010) Clinical use of a system for the automated recording and analysis of electrically evoked compound action potentials (ECAPs) in cochlear implant patients. Acta Otolaryngol 130(6):724-732

19. Wood VH, Bird PA, Giles EC, Baber WJ (2008) Unsuccessful cochlear implantation in two patients with superficial siderosis of the central nervous system. Otol Neurotol 29(5):622-625

20. River Y, Honigman S, Gomori JM, Reches A (1994) Superficial hemosiderosis of the central nervous system. Mov Disord 9(5):559-562
21. Bird PA, Monteath P, Healy L (2010) Successful cochlear implantation in a patient with superficial siderosis of the central nervous system. Otol Neurotol 31(1):177

22. Schievink W, Wasserstein P, Maya M (2016) Intraspinal hemorrhage in spontaneous intracranial hypotension: link to superficial siderosis? Report of 2 cases. J Neurosurg 24:454-456

23. Nadol JB Jr, Adams JC, O'Malley JT (2011) Temporal bone histopathology in a case of sensorineural hearing loss caused by superficial siderosis of the central nervous system and treated by cochlear implantation. Otol Neurotol 32(5):748-755

24. Schvartz-Leyzac KC, Pfingst BE (2018) Assessing the relationship between the electrically evoked compound action potential and speech recognition abilities in bilateral cochlear implant recipients. Ear Hear 39(2):344-358

25. Annual Quality Report (2019) Deutsches Hörzentrum in Hannover 2019

Publisher's Note Springer Nature remains neutral with regard to jurisdictional claims in published maps and institutional affiliations. 